\title{
Resveratrol inhibits phosphorylation within the signal transduction and activator of transcription 3 signaling pathway by activating sirtuin 1 in SW1353 chondrosarcoma cells
}

\author{
HAIDONG JIN* , HUI CHEN* ${ }^{*}$ KEHE YU, JINGDONG ZHANG, BIN LI, NINGYU CAI and JUN PAN \\ Department of Orthopaedic Surgery, The Second Affiliated Hospital of Wenzhou Medical University, \\ Wenzhou, Zhejiang 325000, P.R. China
}

Received May 15, 2015; Accepted June 27, 2016

DOI: $10.3892 / \mathrm{mmr} .2016 .5554$

\begin{abstract}
The present study assessed the mechanism by which resveratrol (Res) inhibits the growth of SW1353 chondrosarcoma cells and examined whether sirtuin 1 (Sirt1) activation affects phosphorylation within the signal transduction and activator of transcription 3 (STAT3) signaling pathway. The present study used SW1353 chondrosarcoma cells in the logarithmic phase of growth (control and treatment groups). The latter group was treated with Res at 25 and $50 \mu \mathrm{mol} / 1$ for $24 \mathrm{~h}$, and cell viability, proliferation and apoptosis were analyzed using the cell counting kit-8 assay, colony counting and Hoechst staining, respectively. The expression levels of caspase-3, cleaved caspase-3, B-cell lymphoma-2 (BCL-2), BCL-2 associated X protein (Bax), STAT3 and phosphorylated (p-)STAT3) were measured by Western blotting. SW1353 cells were transfected with small interfering (si)RNA targeting Sirt1 and the expression levels of Sirt1, STAT3 and p-STAT3 were assessed. Exposure of SW1353 cells to Res reduced cell viability in a dose-dependent manner $(\mathrm{P}<0.01)$. Additionally, cell proliferation was significantly inhibited and the cell nuclei exhibited
\end{abstract}

Correspondence to: Professor Jun Pan, Department of Orthopaedic Surgery, The Second Affiliated Hospital of Wenzhou Medical University, 109 Xueyuan Road (West), Lucheng, Wenzhou, Zhejiang 325000, P.R. China

E-mail: 897898556@qq.com

${ }^{*}$ Contributed equally

Abbreviations: Res, resveratrol; STAT3, signal transduction and activator of transcription 3; Sirt1, sirtuin 1; p-, phosphorylated; FBS, fetal bovine serum; BCL-2, B-cell lymphoma-2; Bax, BCL-2 associated X protein; CCK-8, Cell Counting Kit-8; PBS, phosphate-buffered saline; DMEM, Dulbecco's modified Eagle's medium; siRNA, small interfering RNA; TBST, Tris-buffered saline with Tween-20

Key words: resveratrol, chondrosarcoma, signal transduction and activator of transcription 3 pathway, sirtuin 1 apoptotic characteristics. Cleaved caspase-3, Sirt1 and Bax levels were upregulated. The expression levels of BCL-2 and p-STAT3 were downregulated. Additionally, the BCL-2/Bax ratio was reduced compared with the control group. The total STAT3 level was unaffected. Res treatment activated Sirt1, however, in cells transfected with Sirt1-siRNA, the ability of resveratrol to suppress p-STAT3 expression was compromised. Overall, it was revealed that Res treatment induced apoptosis, inhibited proliferation and affected phosphorylation within the STAT3 signaling pathway by activating Sirt1 in SW1353 chondrosarcoma cells.

\section{Introduction}

Chondrosarcoma is the second most common bone cancer, after osteosarcoma (1). Characteristically, chondrosarcomas contain chondroid cells and a matrix. Surgical excision is the mainstay of treatment, with radiotherapy as an alternative if surgery is contraindicated or if a metastasis requires treatment. Although radiotherapy eliminates tumor cells, normal cells are also harmed. As the side-effects of radiotherapy are therefore serious, more effective anti-chondrosarcoma drugs with minimal toxicity are urgently required. Resveratrol (Res), a dietary phytochemical found in almost 70 plant species, has attracted widespread attention due to the anti-tumor activities it exerts on several types of cancer cell, including hepatocellular carcinoma and gastric cancer cells. However, its effect on chondrosarcoma cells remains unknown. Sirtuin 1 (Sirt1), a histone deacetylase, is widely expressed in various tumor types, including gastric cancer, osteosarcoma and colon cancer. It is well known that Res is an effective agonist of Sirt1 (2).

Signal transduction and activator of transcription 3 (STAT3), a protein constitutively expressed in numerous tissues and cell types, regulates the proliferation, differentiation and apoptosis of normal cells, and maintains normal physiological processes. STAT3 is upregulated in several tumor cells, and it has become increasingly accepted that such upregulation is closely associated with tumorigenesis. Furthermore, previous studies have shown that Res inhibits phosphorylation within the STAT3 signaling pathway in numerous types of tumor cells. However, it remains 
unknown whether Res exerts similar actions in chondrosarcoma cells and, if so, whether Res activates Sirt1 in such cells. The present study assessed the inhibitory effects of Res on chondrosarcoma cells and the underlying mechanisms. In the present study, it was shown that Res induced apoptosis, inhibited cell proliferation and suppressed phosphorylation within the STAT3 signaling pathway by activating Sirt1 in chondrosarcoma cells.

\section{Materials and methods}

Cell culture and reagents. Chondrosarcoma SW1353 cells (Chinese Academy of Life Sciences; Shanghai, China) were cultured in Dulbecco's modified Eagle's medium (DMEM)/F12 medium, supplemented with 10\% (v/v) fetal bovine serum (FBS), penicillin and streptomycin, at $37^{\circ} \mathrm{C}$ in a humidified atmosphere containing $5 \%(\mathrm{v} / \mathrm{v})$ $\mathrm{CO}_{2}$. Res and the Hoechst 33258 reagent were purchased from Sigma-Aldrich (St. Louis, MO, USA). Rabbit antibodies against B-cell lymphoma (BCL)-2 (cat. no. 4223), BCL-2 associated X protein (Bax; cat. no. 5023), caspase 3 (cat. no. 9662), STAT3 (cat. no. 4904), and phosphorylated (p-)STAT3 (cat. no. 9145) were purchased from Cell Signaling Technology, Inc., (Danvers, MA, USA). The cell counting kit (CCK)-8 reagent and Crystal Violet staining solution were purchased from the Beyotime Institute of Biotechnology (Shanghai, China). The Endofectin ${ }^{\mathrm{TM}}$-Plus transfection reagent was purchased from Genecopoeia (Guangzhou, China). A specific Sirt1-small interfering (si) RNA was purchased from GenePharma (Shanghai, China).

CCK-8 assay. SW1353 cells were seeded into 96-well plates at a density of $1 \times 10^{4}$ cells/well and divided into three groups: Blank, Control and Res-treated (2, 5, 10, 25, 50 or $100 \mu \mathrm{mol} / \mathrm{l})$ groups. After $24 \mathrm{~h}$ treatment, $10 \mu \mathrm{l} \mathrm{CCK}-8$ solution was added to each well and the plate was incubated at $37^{\circ} \mathrm{C}$ for $1 \mathrm{~h}$. Cell viability was determined by measuring the absorbance (A) at $450 \mathrm{~nm}$ using a microplate reader (Thermo Fisher Scientific, Inc.). The percentage of proliferative cells were calculated as follows: Relative viability $(\%)=$ $\left(\mathrm{A} 450_{\text {treated }}-\mathrm{A} 450_{\text {blank }}\right) /\left(\mathrm{A} 450_{\text {control }}-\mathrm{A} 450_{\text {blank }}\right) \times 100$.

Colony formation assay. The cells were seeded into 12-well plates at a density of 1,000 cells/well ( $1 \mathrm{ml} /$ well) and divided into a control and a Res-treated group (25 or $50 \mu \mathrm{mol} / \mathrm{l})$. After $24 \mathrm{~h}$ treatment, the plate was incubated in DMEM/F12 medium for 10 days. Following incubation, the cells were fixed in $4 \%$ (v/v) paraformaldehyde for $10 \mathrm{~min}$, washed three times with phosphate-buffered saline (PBS) and stained with crystal violet for $10 \mathrm{~min}$ at $25^{\circ} \mathrm{C}$. The number of visible colonies were then counted and images were captured.

Hoechst 33258 staining. The cells were seeded into 6-well plates at density of $1 \times 10^{5}$ cells $/ \mathrm{ml}(1 \mathrm{ml} / /$ well $)$ and were divided into a control and a Res-treated group ( 25 or $50 \mu \mathrm{mol} / \mathrm{l})$. The cells were incubated with $5 \%(\mathrm{v} / \mathrm{v})$ $\mathrm{CO}_{2}$ at $37^{\circ} \mathrm{C}$ for $24 \mathrm{~h}$, washed three times with PBS, stained with $20 \mu \mathrm{M}$ Hoechst 33258 solution for 20-30 min and were subsequently washed again three times with PBS. Cell

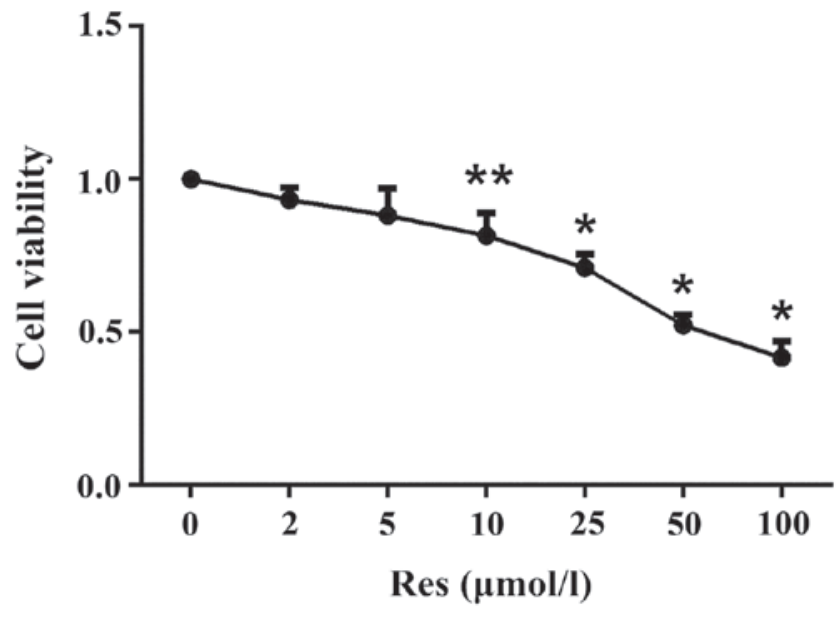

Figure 1. Res reduces the viability of SW1353 cells in a dose-dependent manner. A cell counting kit- 8 assay was used to determine cell viability following the incubation with Res for $24 \mathrm{~h}$. The data are expressed as the mean \pm standard deviation $\left({ }^{*} \mathrm{P}<0.01,{ }^{* *} \mathrm{P}<0.05\right.$ vs. untreated $)$. Res, resveratrol.

morphology was assessed under a fluorescence microscope (Nikon Corporation, Tokyo, Japan).

Sirt1-siRNA transfection. The sequence of chemically modified siRNA was 5'-CGGGAAUCCAAAGGAUAAUTT-3'. The cells were grown overnight and were subsequently transfected with siRNA using Endofectin ${ }^{\mathrm{TM}}$-Plus, according to the manufacturer's protocol. Following incubation for $24 \mathrm{~h}$, the cells were treated with $50 \mu \mathrm{mol} / 1$ Res for $24 \mathrm{~h}$ and cell protein levels were measured by western blotting.

Western blotting. The total protein in was extracted using radioimmunoprecipitation lysis buffer (Beyotime Insititute of Biotechnology). The protein concentrations were determined using a bicinchoninic acid protein assay kit (Sigma-Aldrich, Shanghai, China). A total of $2 \mu \mathrm{g} / \mu \mathrm{l}$ protein was resolved by sodium dodecyl sulfate-polyacrylamide gel electrophoresis and were electroblotted onto nitrocellulose membranes (Beyotime Insititute of Biotechnology). The membranes were subsequently blocked for $2 \mathrm{~h}$ in Tris-buffered saline with $0.5 \%$ Tween-20 (TBST), containing 5\% (w/v) non-fat milk. Following blocking, the membranes were incubated with monoclonal antibodies directed against caspase-3, BCL-2, Bax, STAT3 and p-STAT3 (all 1:1,000) overnight at $4^{\circ} \mathrm{C}$. Following three washes in TBST, the proteins were detected by incubation with horseradish peroxidase-conjugated secondary goat anti-rabbit immunoglobulin G (cat. no. BS13271; 1:5,000) for $2 \mathrm{~h}$. The bands were visualized using enhanced chemiluminescence. A rabbit $\beta$-actin antibody (cat. no. AP0060; 1:3,000; Bioworld Technology, Inc., Nanjing, China) served as a loading control and band densities were quantified using Image Lab version 3.0 software.

Statistical analysis. The data are expressed as the mean \pm standard deviation. All statistical analyses were performed using SPSS version 19.0 (IBM SPSS, Chicago, IL, USA). A one-way analysis of variance and Tukey's post-hoc test were performed. $\mathrm{P}<0.05$ was considered to indicate a statistically significant difference. 


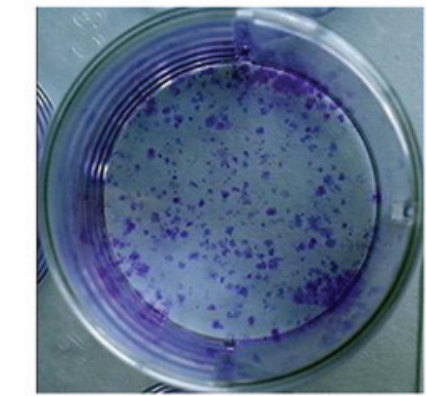

$\operatorname{Res}(\mu \mathrm{mol} / \mathrm{l}) \quad 0$

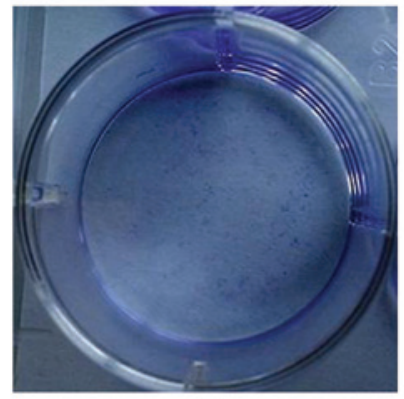

25

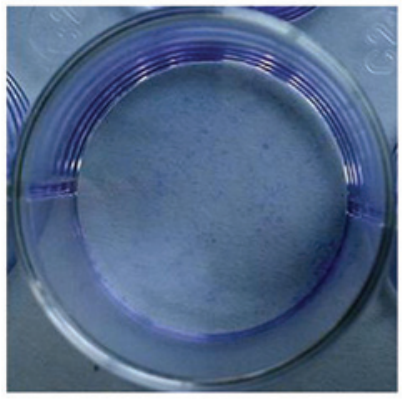

50

Figure 2. Res suppresses SW1353 cell proliferation in a dose-dependent manner. Colony formation assays were performed in triplicate and repeated three times. Representative images are shown. Res, resveratrol.

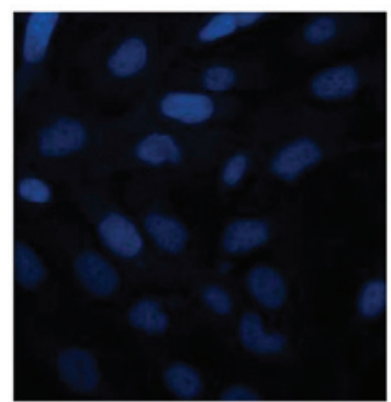

$\operatorname{Res}(\mu \mathrm{mol} / \mathrm{l}) \quad 0$

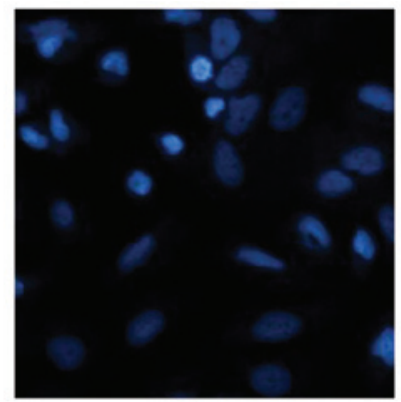

25

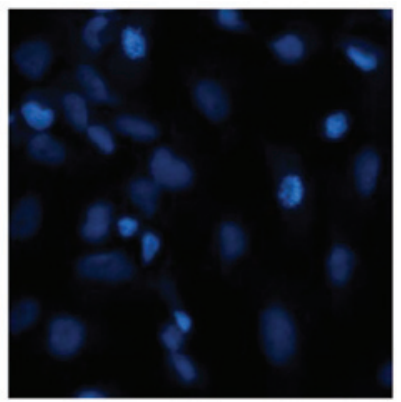

50

Figure 3. Res induces apoptosis in SW1353 cells. Apoptosis was detected via Hoechst 33258 staining and representative images are shown. Res, resveratrol.
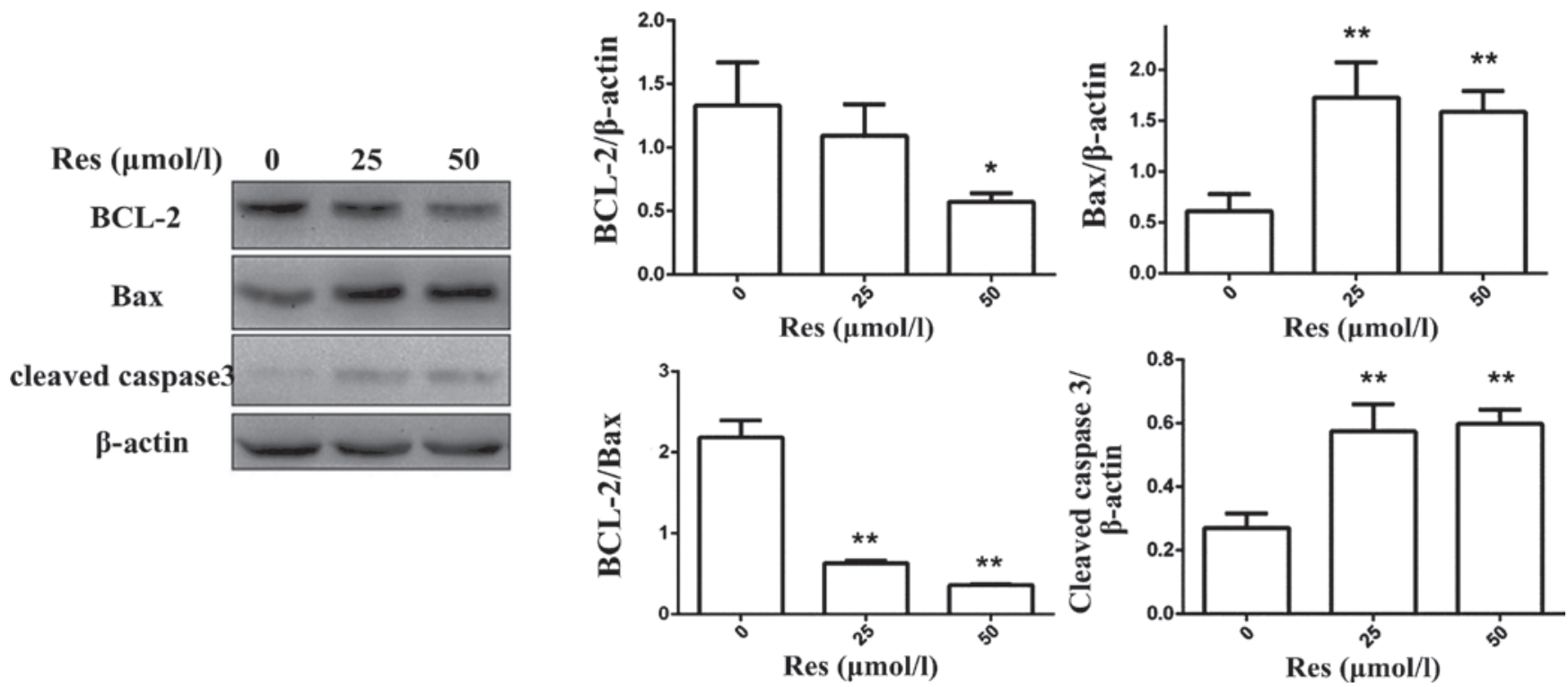

Figure 4. Res increases the expression levels of Bax and cleaved caspase-3, and reduces the expression of Bcl-2, as well as the Bcl-2/Bax ratio. Western blot analysis was performed and the relative expression levels were determined by densitometric analysis of the protein bands. The data are expressed as the mean \pm standard deviation $\left({ }^{*} \mathrm{P}<0.05,{ }^{* * *} \mathrm{P}<0.01\right.$ vs. untreated). Res, resveratrol; Bcl-2, B-cell lymphoma 2; Bax, Bcl-2-associated X protein.

\section{Results}

Res suppresses the proliferation of SW1353 cells in a dose-dependent manner. Cell viability can affect proliferation. Fig. 1 revealed that treatment with Res $(10,25,50$, or $100 \mu \mathrm{mol} / 1$ for $24 \mathrm{~h}$ ) affected the viability of SW1353 cells in a dose-dependent manner. The relative viabilities were $0.8161 \pm 0.0754(\mathrm{P}=0.015)$,
$0.7102 \pm 0.0444(\mathrm{P}=0.0001), 0.5226 \pm 0.0361 \quad(\mathrm{P}=0.0001)$ and $0.4166 \pm 0.0542(\mathrm{P}=0.0001)$. Furthermore, a colony formation assay demonstrated that Res ( 25 or $50 \mu \mathrm{mol} / \mathrm{l})$ significantly reduced cell proliferation compared with the control (Fig. 2).

Res induces the apoptosis of SW1353 cells. The Hoechst 33258 fluorochrome is concentrated in the nucleus of 

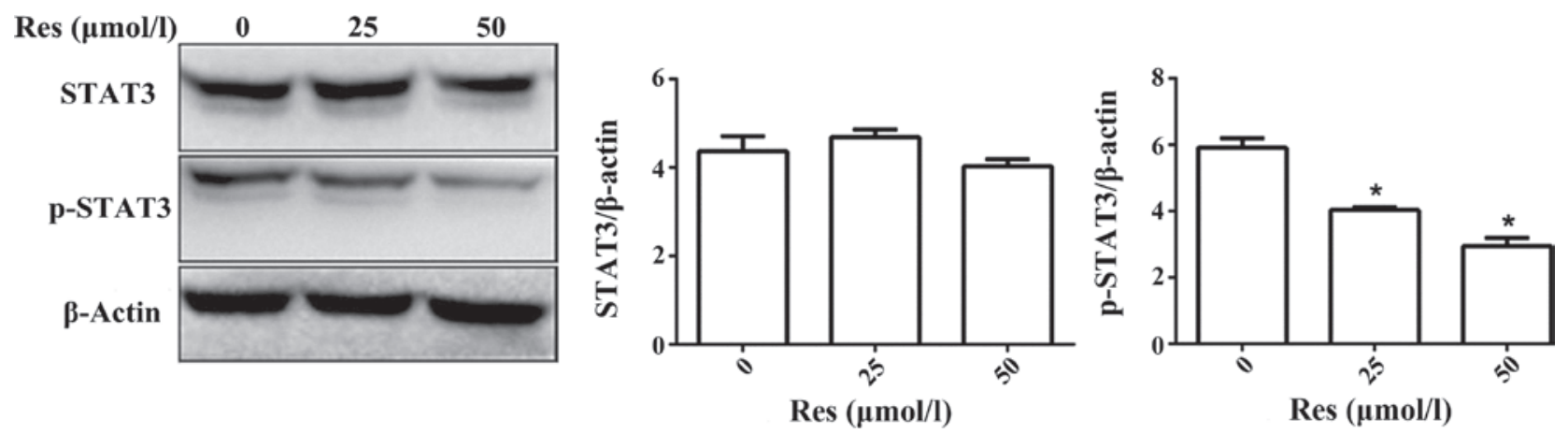

Figure 5. Res reduces the expression of p-STAT3 in SW1353 cells. Western blot analysis was performed to determine the levels of total and p-STAT and relative expression levels were determined by densitometric analysis of protein bands. Values are expressed as the mean \pm standard deviation. "P $<0.05$ vs. untreated. Res, resveratrol; p-STAT, phosphorylated signal transduction and activator of transcription 3.
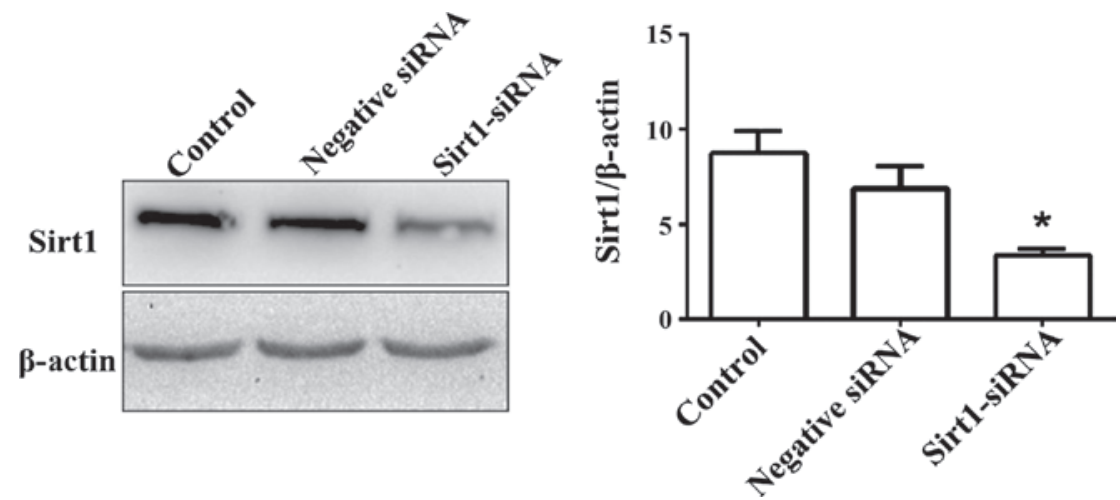

Figure 6. Silencing of Sirt1 in SW1353 cells by transfection with a siRNA-expressing vector. Sirt1 expression levels in control, negative siRNA-transfected and Sirt1-siRNA-transfected groups were determined by western blotting and the relative expression levels were determined by densitometric analysis of protein bands. The data are expressed as the mean \pm standard deviation ("P<0.05 vs. untreated). Sirt1, sirtuin 1; siRNA, small interfering RNA.

$\mathbf{A}$

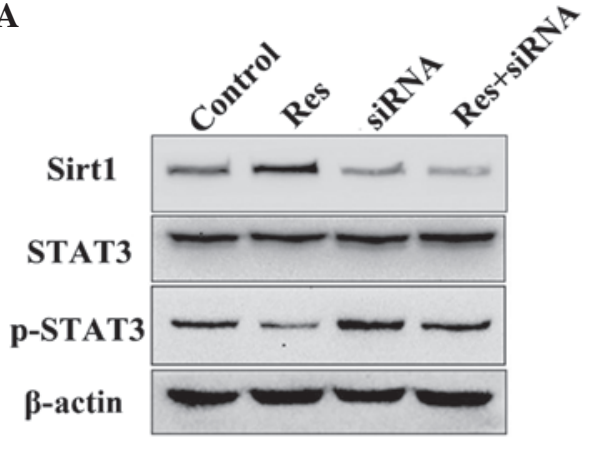

C

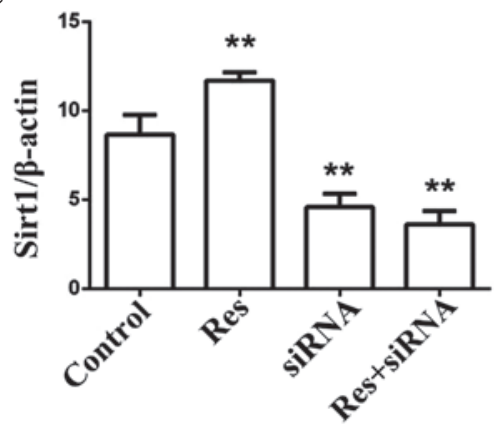

B
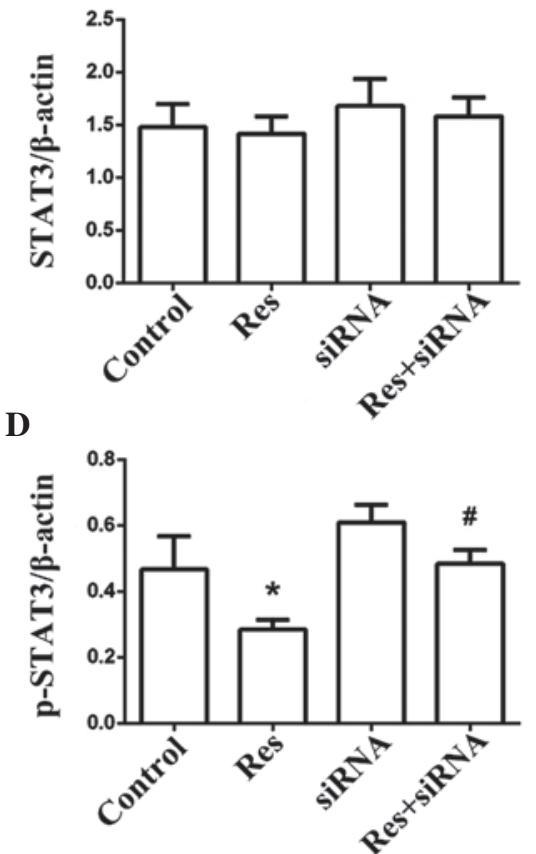

Figure 7. Res activates Sirt1 in SW1353 cells and its suppressive action on the protein expression of p-STAT3 via the activation of Sirt1 was weakened in Sirt1-siRNA cells,. (A) The expression levels of Sirt1, total STAT3, and p-STAT3 in control, Res-treated, Sirt1-siRNA-transfected and Res-treated + Sirt1siRNA-transfected groups were determined via Western blotting. (B-D) The relative expression levels are shown in histograms. The data are expressed as the mean \pm standard deviation ( ${ }^{*} \mathrm{P}<0.05,{ }^{* *} \mathrm{P}<0.01$ compared with the control group; ${ }^{*} \mathrm{P}<0.05$ compared with the Res group). . Sirt1, sirtuin 1 ; siRNA, small interfering RNA; p-, phosphorylated; Res, resveratrol; STAT, signal transduction and activator of transcription 3. 
apoptotic cells. As shown in Fig. 3, the rate of apoptosis of Res-treated cells was significantly higher compared with that of controls.

Res increases the expression levels of Bax and cleaved caspase 3, and reduces the expression levels of Bcl-2 and the Bcl-2/Bax ratio. Bcl-2, Bax, and cleaved caspase-3 serve important roles in the mitochondrial pathway of apoptosis. Res $(25$ or $50 \mu \mathrm{mol} / \mathrm{l})$ significantly upregulated the expression levels of Bax and cleaved caspase-3 ( $\mathrm{P}<0.01$; Fig. 4). In addition, $\operatorname{Res}(50 \mu \mathrm{mol} / \mathrm{l})$ reduced the expression of $\mathrm{Bcl}-2(\mathrm{P}<0.01)$, but only at $50 \mu \mathrm{mol} / 1$. Res (25 or $50 \mu \mathrm{mol} / \mathrm{l})$ reduced the Bcl-2/Bax ratio (Fig. 4E).

Res reduces the expression of p-STAT3 in SW1353 cells. Western blotting was performed to measure the expression levels of p-STAT3 and STAT3 in SW1353 cells treated with Res $(0,25$ or $50 \mathrm{mmol} / \mathrm{l})$. p-STAT3 was downregulated in a Res-dependent manner $(\mathrm{P}<0.01)$, however, the total STAT3 level did not significantly change (Fig. 5).

Effect of Sirtl downregulation on the STAT3 signaling pathway. Sirt1-siRNA significantly suppressed Sirt1 protein expression compared with its expression in the control or negative-siRNA-transfected group $(\mathrm{P}<0.01$; Fig. 6). The expression levels of Sirt1, p-STAT3, total STAT3 and $\beta$-actin were assessed in SW1353 cells (control, Res-treated, siRNA and Res + siRNA). Res $(50 \mu \mathrm{mol} / \mathrm{l})$ activated the expression of Sirt1 $(\mathrm{P}<0.05)$, but not after siRNA transfection. Treatment with Res $(50 \mu \mathrm{mol} / \mathrm{l})$ suppressed the expression of p-STAT3 and caused no significant effect on the total STAT3 levels. p-STAT3 expression was not inhibited in the siRNA or Res+siRNA group $(\mathrm{P}<0.01)$, no significant difference was identified between the total STAT3 levels in the siRNA and Res + siRNA group ( $\mathrm{P}=0.14$; Fig. 7$)$.

\section{Discussion}

The effects of res, which was first discovered in 1940, on different types of tumor cells have been studied to varying degrees. In hepatocellular carcinoma, gastric cancer and breast cancer cells, Res inhibits cell proliferation, induces apoptosis and inhibits the cell cycle (3-5). In HepG2 hepatocellular carcinoma cells, Res induces apoptosis by activating p53 and upregulating the expression levels of Bax and p21 (6). In addition, in cutaneous carcinoma cells, Res activates mitochondrial proteins, including caspases-3, -8 and -9 , and poly (ADP-ribose) polymerase, triggers the release of cytochrome $c$, thus activating Bax, and suppresses the expression of Bcl-2. Apoptosis follows this process (7).

The present study found that Res reduced the proliferation and induced the apoptosis of SW1353 cells. Res increased the expression levels of Bax and cleaved caspase-3, and downregulated the expression of $\mathrm{Bcl}-2$. Res also significantly reduced the Bcl-2/Bax ratio, indicating that Res can inhibit cell proliferation and induce apoptosis via the mitochondrial pathway.

STAT3, expressed by numerous cells and tissues, is an important member of the STAT protein family. Continuous STAT3 activation triggers the ingravescence of tumor cells and tissues, indicating that the STAT3 signaling pathway is intimately involved in tumor cell proliferation and apoptosis (5). STAT3 phosphorylation upregulates the expression of apoptosis-inhibitory proteins, including Bcl-2, Bcl-xL, Mcl-1, and Survivin, and downregulates the expression of Bax. STAT3 also activates the expression of cyclin D1, cell division cycle 2, c-myc, cyclinB1, c-jun and c-fos, which may trigger malignant proliferation (8-11). The present study found that Res suppressed phosphorylation of STAT3, showing that the inhibitory effects of Res on chondrosarcoma proliferation were partly attributable to the phosphorylation of STAT3.

Sirt1, a member of the class III nicotinamide adenine (+)-dependent histone deacetylase Sirt family, is involved in various physiological processes, including differentiation, apoptosis and metabolism (12). Res is an effective Sirt1 agonist and the covalent binding of Res to Sirt1 alters the conformation of Sirt1, increasing the affinity of the protein for its substrate (13). Numerous previous reports have shown that Sirtl gene knockdown induces the expression of STAT3 in fibroblast cells and that Sirt1 upregulation inhibits acetylation within the STAT3 signaling pathway of keratinocytes (14-16). These data indicated that the STAT3 signaling pathway is regulated by Sirt1. The present study found that Res induced Sirtl expression and suppressed phosphorylation within the STAT3 signaling pathway. Additionally, STAT3 phosphorylation was significantly inhibited by Res, however, this was negated by Sirt1-siRNA. Taken together, the data revealed that Res suppresses phosphorylation within the STAT3 signaling pathway by activating Sirt1 in chondrosarcoma cells.

In conclusion, res, a natural anti-tumor material, exerts diverse anti-tumor effects, including induction of apoptosis, inhibition of cell proliferation and suppression of phosphorylation within the STAT3 signaling pathway by activating Sirt1 in chondrosarcoma cells. However, the mechanism by which Sirt1 affects phosphorylation within the STAT3 pathway remains to be elucidated.

\section{Acknowledgements}

The authors would like to thank Mr. L.Y. Cai, Mr. N. Majid and Mr. L. Chen (Wenzhou Medical University, Wenzhou, China) for their comments and advice. The present study was supported by the Zhejiang Provincial Medical Science and Technology Project (no. 2014RCA017).

\section{References}

1. Liang W, Li X, Li Y, Li C, Gao B, Gan H, Li S, Shen J, Kang J, Ding $S$, et al: Gallic acid induces apoptosis and inhibits cell migration by upregulating miR-518b in SW1353 human chondrosarcoma cells. Int J Oncol 44: 91-98, 2014.

2. Villalba JM and Alcaín FJ: Sirtuin activators and inhibitors. Biofactors 38: 349-359, 2012.

3. Yang Q, Wang B, Zang W, Wang X, Liu Z, Li W and Jia J: Resveratrol inhibits the growth of gastric cancer by inducing G1 phase arrest and senescence in a Sirt1-dependent manner. PloS One 8: e70627, 2013.

4. Mezzanotte L, An N, Mol IM, Löwik CW and Kaijzel EL: A new multicolor bioluminescence imaging platform to investigate $\mathrm{NF}-\kappa \mathrm{B}$ Qactivity and apoptosis in human breast cancer cells. PloS One 9: e85550, 2014.

5. Carter LG, D'Orazio JA and Pearson KJ: Resveratrol and cancer: Focus on in vivo evidence. Endocr Relat Cancer 21: R209-R225, 2014. 
6. Kuo PL, Chiang LC and Lin CC: Resveratrol-induced apoptosis is mediated by p53-dependent pathway in Hep G2 cells. Life Sci 72: 23-34, 2002.

7. Kalra N, Roy P, Prasad S and Shukla Y: Resveratrol induces apoptosis involving mitochondrial pathways in mouse skin tumorigenesis. Life Sci 82: 348-358, 2008.

8. Yu H, Pardoll D and Jove R: STATs in cancer inflammation and immunity: A leading role for STAT3. Nat Rev Cancer 9: 798-809, 2009.

9. Germain D and Frank DA: Targeting the cytoplasmic and nuclear functions of signal transducers and activators of transcription 3 for cancer therapy. Clin Cancer Res 13: 5665-5669, 2007.

10. Tebbutt NC, Giraud AS, Inglese M, Jenkins B, Waring P, Clay FJ, Malki S, Alderman BM, Grail D, Hollande F, et al: Reciprocal regulation of gastrointestinal homeostasis by SHP2 and STAT-mediated trefoil gene activation in gp130 mutant mice. Nat Med 8: 1089-1097, 2002.

11. Ranger JJ, Levy DE, Shahalizadeh S, Hallett M and Muller WJ: Identification of a Stat3-dependent transcription regulatory network involved in metastatic progression. Cancer Res 69 : 6823-6830, 2009.
12. Deng CX: SIRT1, is it a tumor promoter or tumor suppressor? Int J Biol Sci 5: 147-152, 2009.

13. Borra MT, Smith BC and Denu JM: Mechanism of human SIRT1 activation by resveratrol. J Biol Chem 280: 17187-17195, 2005.

14. Bernier M,Paul RK, Martin-Montalvo A,Scheibye-Knudsen M, Song S, He HJ, Armour SM, Hubbard BP, Bohr VA Wang L, et al: Negative regulation of STAT3 protein-mediated cellular respiration by SIRT1 protein. J Biol Chem 286: 19270-19279, 2011.

15. Sestito R, Madonna S, Scarponi C, Cianfarani F, Failla CM, Cavani A, Girolomoni G and Albanesi C: STAT3-dependent effects of IL-22 in human keratinocytes are counterregulated by sirtuin 1 through a direct inhibition of STAT3 acetylation. FASEB J 25: 916-927, 2011.

16. Li Y, Zhu W, Li J, Liu M and Wei M: Resveratrol suppresses the STAT3 signaling pathway and inhibits proliferation of high glucose-exposed HepG2 cells partly through SIRT1. Oncol Rep 30: 2820-2828, 2013. 